\title{
Health Food and Traditional Chinese Medicine in China
}

\author{
Hou H, Li S and Ye Z
}

Institute of Chinese Materia Medica, China Academy of Chinese Medical Sciences, Beijing, 100700, China

*Corresponding author: Ye Z, Institute of Chinese Materia Medica, China Academy of Chinese Medical Sciences, Beijing, 100700, China, Fax: +86 84212805, Tel: +86 84212805, E-mail: yezuguang@sina.com

Citation: Hou H, Li S, Ye Z (2016) Health Food and Traditional Chinese Medicine in China. J Nutr Health Sci 3(3): 301. doi: 10.15744/2393-9060.3.301

Received Date: May 25, 2016 Accepted Date: August 22, 2016 Published Date: August 24, 2016

\begin{abstract}
Like a drug for a purpose of treating or preventing a disease, a health food has to be scientifically evaluation and officially approved before it legally is marketed in China. Health foods in China are characterized by involvement of Chinese herbs in them. Traditional Chinese Medicine (TCM) theory consists of both health care and disease's diagnosis and treatment, and therefore, heath care plays an important role in TCM system. Historically, TCM has evolved and derived from food therapy. Some TCM raw materials have two sides: one is used for a medical drug, and the other side is for a health food. Formulation of the health food consisting of TCM materials is directed by TCM theory and knowledge. The health foods with TCM as raw materials account for more than $50 \%$ of all the health foods marketed in China. In sum, health food has a close relationship with TCM.
\end{abstract}

Keywords: Traditional Chinese Medicine; Health food; Health care; Food therapy; Regulation

\section{Introduction}

Health food refers to the food which has health care function, or can supplement the human body nutritional needs such as vitamins and minerals. And it helps people to regulate or improve the physiological function. However it is by no mean used for treatment of the disease. The most important thing must be borne in mind that it must not produce any acute, sub-acute or chronic damage to the body [1]. In China, there are 2 categories of health foods: one is functional food, which claims a certain improvement of physiological functions, and the other is dietary supplement, which is a kind of nutritional supplement to human body. Health food can be an adjunctive remedy to the drug therapy or other medical treatment, and it is mainly for sub-health population. With the improvement of Chinese living standards, the people have higher and higher demands for the life quality, which makes the market of health food become one of the fastest growing industries. In China, all the health food needs the evaluation and approval by China Food and Drug Administration (CFDA) before its legal production and marketing. CFDA has issued a series of regulation and technical requirements to guide the $\mathrm{R} \& \mathrm{D}$, evaluation and approval of health food.

Heath care and maintaining the human body in good shape is an indispensable part of TCM theory and practice. Consequently, $\mathrm{R} \& \mathrm{D}$ of a functional food is closely correlated with Traditional Chinese Medicine (TCM). Therefore, most of the China-made functional foods are characterized partially or totally by Chinese herbs, which has made them different from those functional foods produced by other countries $[2,3]$.

This paper essentially introduces the management of functional food focusing on its technical evaluation and official approval procedure in China, and also reviews the relationship between TCM and the health food.

\section{Evaluation and approval of health food and its technical requirements}

\section{Formation and perfection of law and regulations on health food}

There had been no special regulatory documents on the health food in China until 1995 when Food Hygiene Law of the People's Republic of China was promulgated by the National People's Congress, and since then, the legal status of health food has been established in China. With guidance of the law, "Regulation for the Registration of Health Food" was issued by Ministry of Public Health, which specified in details the evaluation and approval, registration, supervision and management of health food. In 2003, the administrative office of health food and its authorization were transferred to CFDA from Ministry of Public Health. Later on, CFDA issued a revised vision of "Regulation on Registration for Health Food". Recently, with more attention paid to food safety, "Food Hygiene Law of the People's Republic of China” was revised, and then, "Food Safety Law of the People's Republic of China was promulgated by National People's Congress in 2009 and in 2015, respectively, both of which emphasize the safety of food, 
and furthermore, require the strict management of health food in term of the food safety. In addition to the acts and regulations mentioned-above, a series of technical requirements and guidelines in details for the registration and approval of the health food have been issued by administrative authorities concerned, which contains a special regulation on TCM raw materials for health food, which clearly points out what Chinese herbs can be used as raw materials of health food, and what Chinese herbs can't be used.

\section{The evaluation and approval system of health food}

CFDA is responsible for the evaluation and the approval of health food's registration for marketing. Actually, its technical evaluation is carried out by a professional body, Center for Evaluation of Health food and Cosmetics, which is directly under CFDA. Both outside experts and the Center work together to share the evaluation in practice, where the outside experts play a crucial role in technique evaluation. There is an outside experts committee consisting of hundreds of experts with various disciplines. The experts are randomly selected for a given evaluation.

There are two phases in the evaluation and approval system of health food: the first phase is preliminary evaluation conducted by a local branch of CFDA, including on-site inspection, reviewing all the experimental records, verifying the sample's quality in a lab and so on; the second phase is the final evaluation at the state level, following a successful preliminary evaluation in the local office. The final evaluation is fulfilled by the outside experts as well as the Center for Evaluation of Heath Food and Cosmetics. In general, the application dossier is firstly submitted to the local branch of CFDA for the preliminary evaluation, and after passing the preliminary evaluation, the application goes up to CFDA for the final evaluation, where a final decision is issued to the applicant, regardless of the approval or not (Figure 1).
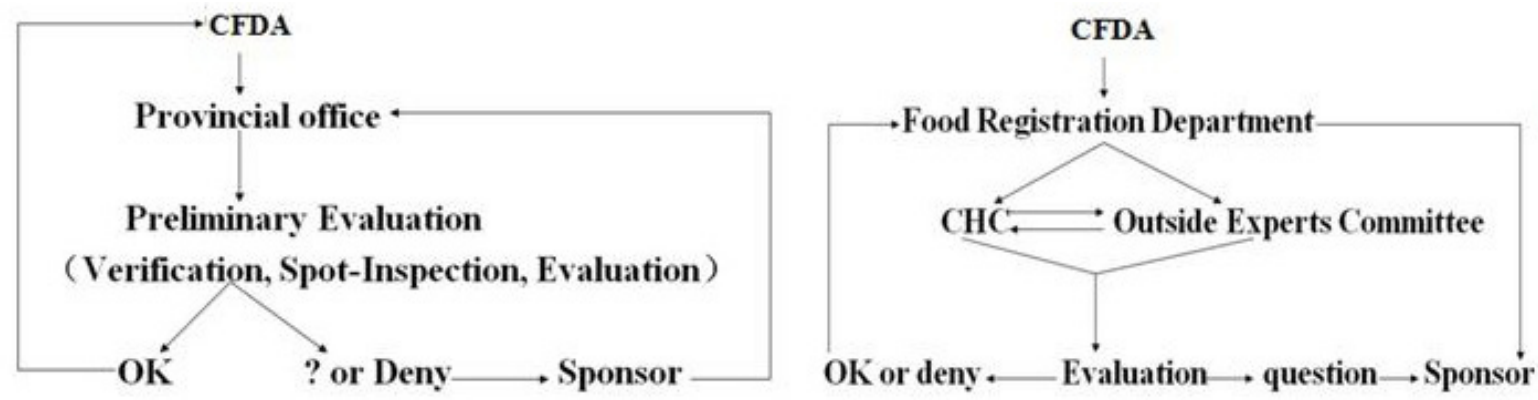

Figure 1: Procedure of evaluation of health food. A Preliminary evaluation at a provincial level; B Final evaluation at a state level; CHC Center for Evaluation of Health food and Cosmetics

\section{The technical requirements of the health food's $R \& D$}

Pre-market researches of health food involve the functions tests (laboratory research and clinical research), toxicological tests for safety evaluation and quality control of the product [4]. Except the studies finished by an applicant himself, a set of confirmatory tests mentioned-above on health food must be conducted by a qualified institution officially-appointed by CFDA, which will present all the reports of the tests to CFDA for the evaluation. Currently, there are only 27 kinds of functions or health claims for the health food in China, such as stimulation of immune function, improvement of sleep, skin-care and so on. A set of specified experimental data are needed for a certain functional food, for example, a health food applying for stimulating immune function is required to conduct a set of immunological tests to prove its health claim, while, for a function of increasing bone density, other tests related to bone density are required. In order to standardize the tests required for a given functional food, the Ministry of Public Health published the relevant guidelines for experiments needed to be done for a certain functional food in 2003 [5]. Therefore, an applicant has to complete the tests particular for the health claim he selects, based on the guidelines by the authorities (Table 1). In addition to the above 27 kinds of functions, CFDA also encourages the development of new functions of health food, which certainly need to provide a large number of research data to scientifically underlie the new function. The functional tests can be divided into three categories: the functions where only animal experiments in the lab are needed, for example, the function for promoting immunity or increasing bone density; while the functions where human trials but not animal experiments are needed, such as skin care and beauty; for some function foods, both animal experiment and human trials are needed for some functions, for example, the function for decreasing blood-lipid, blood sugar and blood pressure. Clinical studies need to be done in the qualified hospitals and the subjects include 50 treated cases and 50 cases vehicle control. Only functional food is required to do functional test in lab or in hospital for clinical trial, while dietary supplements don't need to do functional test, such as vitamin supplement and minerals supplement (calcium, zinc, selenium, etc.).

From administrative point of view, health food is classified as one kind of food, and its safety is especially underlined. The toxicity tests include acute toxicity, chronic toxicity (30 days feeding test or 90 days feeding test), mutation detection and reproductive toxicity, all of which should conducted in the qualified labs with qualified animals. Like Chemistry, Manufacture and Control (CMC) for a new drug application, CMC-like data such as producing technological study including pilot study, quality control 
standards and the stability tests are submitted to CFDA for the evaluation and the registration. After the completion of the above tests, all the tests reports and application materials should be submitted to CFDA to experience two phases of the evaluation procedures mentioned previously.

\begin{tabular}{|c|c|c|}
\hline Classification & Number & Functions \\
\hline $\begin{array}{c}\text { only asked to do } \\
\text { animal experiments }\end{array}$ & 7 & $\begin{array}{c}\text { promote immunity, help sleeping: insomnia, fatigue-relieving, increase tolerence to hypoxia, } \\
\text { prevent from radiation, protect liver from damage induced by chemicals, bone density }\end{array}$ \\
\hline $\begin{array}{c}\text { only asked to do } \\
\text { human trials }\end{array}$ & 5 & eyesight, acne, chloasma, skin-care: maintain skin moisture \\
\hline $\begin{array}{c}\text { asked to do both } \\
\text { animal experiment } \\
\text { and human trials }\end{array}$ & 15 & $\begin{array}{c}\text { decrease blood-lipid, decrease blood sugar, anti-oxidization, improve memory, excrete lead(Pb) } \\
\text { from body, laryngitis and pharyngitis, normalize blood pressure, increase lactation, for obesity, } \\
\text { promote growth, nutritional anemia, moistening the intestine and relieving constipation, mu- } \\
\text { cous membrane of stomach, disorder of intestinal flora, increase digest function }\end{array}$ \\
\hline
\end{tabular}

Table 1: 27 kinds of health food and classification

In addition, the production of health food is implemented following Good Manufacturing Practice (GMP) standard. In order to promote GMP practice, the Ministry of Public Health issued "Good Manufacturing Practices of Health Food" in 1998, and the guidelines for health food GMP practice was also issued, which ensure the quality and safety of health food [6].

\section{TCM roles in the $\mathrm{R} \& \mathrm{D}$ of health food}

\section{TCM is one of raw material sources of Chinese health food}

Maintaining physiological functions of human body in balance status is a key principle of TCM, and it says that causally, a given disease, which manifests itself in a limited location of the body like blood pressure, blood sugar, tumor and so on, stems from unbalance of physiological functions of the body as a whole. First of all, TCM treatment emphasizes more regulating the body from unbalance into balance status than overcoming the local problems. Furthermore, the TCM physicians who undertake medical interference in sub-health or pre-disease stage, is much better than those who treats patient only following manifestation of a disease or its symptoms. Hence, TCM pays more attention to health care and sub-health status, which shares similarities with the definition and the claims of the health food. Apart from TCM theory associated with health food, a large number of TCM raw materials are frequently used for maintaining and keeping the body in healthy condition, and these TCM materials are not only used for treating a disease, but also adopted for functional food. Legally, some of the TCM raw materials also are the raw materials of the health food. Generally, the raw materials of health food consist of following categories: vitamins, some minerals, some natural products, Chinese herbs, and other edible materials. According to statistics, the formulae of the health food containing the TCM raw materials account for more than $50 \%$ of all the approved health foods in China. Thus, TCM has contributed greatly to the raw materials of health food. However, based on safety concerns, not all Chinese herbs are used as the raw materials of health food. In order to clearly define the TCM raw materials that can be adopted as the raw materials in the health food, the Ministry of Public Health issued the notice on further standardizing management of the raw materials of health food in February 2002 ([2002] No. 51, hereinafter referred to as "NO.51 document"), which attaches three documents: Appendix 1(a list of the TCM materials that can be used as both ordinary food and health food), Appendix 2(a list of the TCM materials that are used only as health food but not ordinary food), and Appendix 3(a list of the TCM materials that are definitely not allowed for the health food or ordinary food). Appendix 1 includes 87 Chinese herbs, such as dioscoreae rhizome, crataegi fructus, glycyrrhizae radix et rhizome, asini corii colla, houttuyniae herba, lycii fructus, gardeniae fructus, poria, puerariae lobatae radix, ziziphi spinosae semen, etc. Appendix 2 includes 114 Chinese herbs, such as, ginseng radix et rhizome, ligustri lucidi fructus, salviae miltiorrhizae radix et rhizome, gastrodiae rhizome, angelicae sinensis radix, rhodiolae crenulatae radix et rhizo, panacis quinquefolii radix, codonopsis radix, leonuri herba, epimedii folium, etc. And Appendix 3 includes 59 that are recognized as toxic materials, such as, aconiti kusnezoffii radix, strychni semen, crotonis fructus, kansui radix, euphorbiae ebracteolatae radix, daturae flos, pharbitidis semen, periplocae cortex, mylabris, Tripterygium wilfordii, etc.

Recently, for the purpose of safety, Rheum palmatum, Scutellaria baicalensis, Coptis chinensis, Phellodendron amurense and Centipeda minima are also prohibited as raw materials of health food. Thus, use of Chinese herbs as the ingredients of a health food is standardized to avoid its misuse. In addition, according to the relevant regulations, the first and the second class national protected animals, plant and their products are not allowed to be used as the raw materials of health food. But if the secondary class national protected animals and plant are cultivated, they can be allowed for the raw materials of health food [7].

\section{How to scientifically use TCM raw materials in health food?}

Because consumers easily get access to the health food at will, its safety has been concerned. Also, the same attention is paid to the safety of the health food with TCM ingredients, especially the orally-taken amount of the health food and the administrative duration. "Pharmacopoeia of the People's Republic of China" (hereinafter referred to as "Pharmacopoeia") specifies the recommended dosage range of TCM as raw materials. When TCM raw materials are planned to be used as ingredients of a health food, the dosage of TCM raw materials in the formula should follow the dose-recommendation of "Pharmacopoeia". For sake 
of safety to consumers, Chinese herbs ingredient dose in a health product is generally selected to be around the lower limit of recommended dose of "Pharmacopoeia". If the dosage of Chinese herbs in health food more than the limit of recommendation of "Pharmacopoeia", it will need more scientific data to prove its safety [7].

TCM for medical purpose or health care is characterized by a combination of two or more TCM raw materials in a prescription or a formula. Therefore, compatibility of serial TCM raw materials in a combination is an important part of TCM theory and practice in health care. In health care practice of TCM, the preparation with Chinese herbs multi-ingredients is commonly found. As a result, TCM theory on compatibility of the Chinese herbs combination has been developed and Chinese herbs combination experience is also summarized, such as 7 kinds of interaction nature of Chinese herbs combination, including synergism, antagonism, and addition, and the Eighteen Incompatible medicaments, the Nineteen Medicaments of Mutual Restraint [8]. Consequently, when a health food consisting of TCM materials combination is researched and developed into a commercial product, its formulation always follows the compatibility theory and practical experience of Chinese herbs combination. Meanwhile, its formulation is also based on modern pharmacological evidence and scientific principle. If so, health function will be potentiated and side effect will be minimized. It is a key aspect that the producing process of a health food with TCM as raw materials usually adopts the traditional technology, which in general is the process of water extraction. But sometimes, in order to obtain the lipid-soluble effective components, alcohol extraction is occasionally used. For safe consideration, the production of bacterial fermentation is not allowed for processing TCM materials.

In summary, there is a close relationship between TCM and health food in theory and practice. But there is a lot of room to be improved in the research, development, registration, industrialization and internationalization of health food with TCM as raw materials.

\section{Acknowledgement}

This research was supported by National science and technology major projects: Safety testing technology and Standard of new drug of TCM ( 2014 zx09304307-001)and ICMM project grant (ZXKT15005).

\section{References}

1. State Food and Drug Administration (2007) The announcement about named regulations of health food by the State Food and Drug Administration. Chin Food Hygien 19: 374.

2. Hu J, Xu Q (1998) The research and development opportunity of health food using TCM as raw materials. Chin J Inform Tradit Chin Med 4: 27-8.

3. Zhang X, Ye Z (2003) Annual work report of approval and research center of FDA in 2000 and 2001. Chin J New Drug 1: 4-8.

4. Ye Z, Zhang G (2014) Progress of safety evaluation of traditional Chinese medicines and its strategy in further studies. Chin J Exp Tradit Med Formul 16: 1-6.

5. Inspection and technical specifications of evaluation of health food issued by the Ministry of Public Health (2003). Chin J Food Hygiene 3: 269.

6. Xu K (2004) Implementation guidelines of manufacturing practices of health food. Press Distribution of Chemical Industry.

7. Dang Y, Xiao P, Yang X (1998) Comments on How to Combine Chinese Medicine with Modern Functional Food. Lishizhen Med Material Med Res 6: 6-7.

8. Li Y, Gao J (2007) Health food? Traditional Chinese Medicine?-- more and more the formula of health food formula is the same as of TCM. Capital Med 9: 22-3.

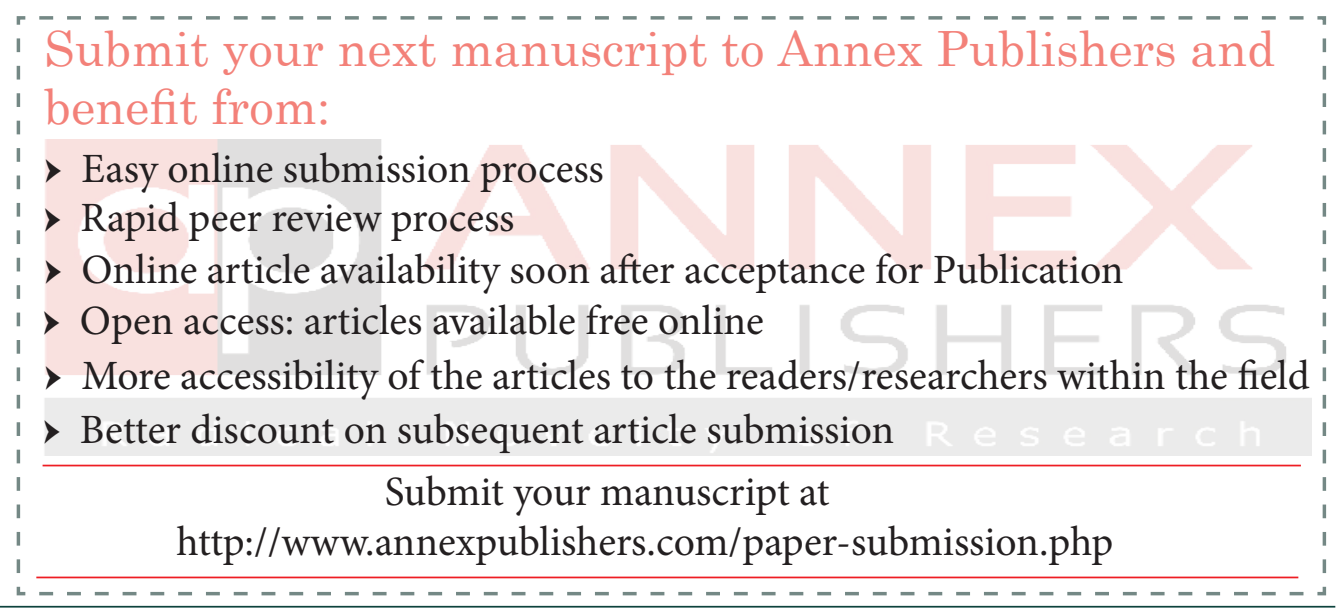

